JGSP 13 (2009) 9-24

\title{
SOME SOLUTIONS WITH TORSION IN CHERN-SIMONS GRAVITY AND OBSERVABLE EFFECTS
}

\author{
FABRIZIO CANFORA
}

Communicated by Gaetano Vilasi

\begin{abstract}
It is shown that in five dimensional Einstein-Gauss-Bonnet theories exact vacuum solutions with non-vanishing torsion can be constructed.

Some possible observational effects related to neutrino oscillations are pointed out. In the theory of continuum media (in which suitable defects can be described by localized non vanishing torsion) "the gravitational intuition" is a rather useful tool to describe the physical effects of such defects. A possible astrophysical application is shortly described.
\end{abstract}

\section{Introduction}

Finding the correct quantum theory of gravity is a major problem in theoretical physics. Although there are very strong proposals such as superstring theory and loop quantum gravity, the final answer has not been found yet. Thus, it is worth to explore new possibilities. Among the many proposals available in the literature, the possibility to have gravitational actions different from the Einstein-Hilbert one and with non vanishing torsion is worth to be explored. The most natural generalizations of the Einstein-Hilbert action are the so called Lovelock action [1]. Lovelock actions are the most general covariant actions leading to second order equation for the metric (it is a very difficult task to provide one with a complete list of references on the subject; a nice review on the black hole solutions is [2]). Unlike the four dimensional case in which torsion vanishes, in higher dimensional Lovelock theories torsion may represent propagating degrees of freedom [3], [4]. However, up to now the possible dynamical role of torsion has not been taken into account too seriously.

In this paper an intriguing analogy between BPS states in QFT and gravity with torsion will be explored. This analogy allows to find interesting solutions in five dimensional Einstein-Gauss-Bonnet gravity in which the torsion looks like topological excitations and may improve the stability of torsion-free solutions. Fur- 
thermore, torsion could led to observable effects both in high energy physics and in solid state physics.

The paper is organized as follows: in the second section a very short review of BPS states in field theory is presented. In the third section, a general introduction to gravitational models with torsion is described. In the fourth section the analogy of BPS states in field theory and gravitational model with torsion is presented and how to construct solutions of Chern-Simons gravitational theories with torsion starting from solutions without torsion is discussed. In the fifth section, some observable effects in high energy physics and in solid state physics are discussed. Eventually, some conclusions are drawn.

\section{Very Short Review on BPS in Field Theory}

In this section it will be shortly resumed BPS states in field theory with attention on vortices. Because of the enormous number of papers on this subject, it is a hopeless task to provide with a complete list of references, the main reference on which is based the present paper is the detailed reviews [5] and [6].

A suitable gauge theoretical action able to give rise to vortex-like solutions is

$$
S=\int \mathrm{d}^{4} x\left[\frac{1}{4} F_{\mu \nu} F^{\mu \nu}+\left|D_{\mu} \phi\right|^{2}-\frac{\lambda}{4}\left(|\phi|^{2}-v^{2}\right)^{2}\right]
$$

where $F_{\mu \nu}$ is the field strength of an Abelian U(1) connection, $\phi$ is a complex $U(1)$-charged scalar field (whose charge is $e$ ) with the standard symmetry breaking potential whose minima are of the form

$$
\phi_{v a c}=v \exp (\mathrm{i} \alpha)
$$

where $\alpha$ is a real phase. The energy of a static vortex configuration (in which the vortex is along the third axis) is

$$
E_{A H}=\int \mathrm{d}^{2} x\left[\frac{1}{2} B^{2}+|\vec{D} \phi|^{2}+\frac{e^{2}}{2}\left(|\phi|^{2}-v^{2}\right)^{2}\right] \geq v^{2}\left|\Phi_{B}\right|
$$

with $\Phi_{B}$ being the magnetic flux. For any finite energy solution $\phi$ approaches the vacuum manifold at infinity. Therefore the finite energy solutions are classified by their winding number $N$ (also called vorticity) which counts the number of times the phase of $\phi$ winds around the spatial circle at infinity

$$
\phi \underset{r \rightarrow \infty}{\rightarrow} v \exp (\mathrm{i} N \theta) .
$$


The above winding number labels homotopy classes. The vorticity is related to the magnetic flux $\Phi_{B}$ since

$$
\{\vec{D} \phi \underset{r \rightarrow \infty}{\rightarrow} 0\} \Rightarrow e A_{i} \sim-\mathrm{i} \partial_{i} \log \phi=N \partial_{i} \theta \Rightarrow \Phi_{B}=2 \pi N .
$$

The Bogomol'nyi equations can be deduced assuming that the bound is saturated:

$$
B \mp e\left(|\phi|^{2}-v^{2}\right)=0, \quad\left(D_{1} \pm \mathrm{i} D_{2}\right) \phi=0 .
$$

Such equations (which are linear relations among the magnetic part of the curvature and the square of the Higgs field) will be very important to formulate the analogy and to establish a dictionary between "BPS gauge theoretical objects" and "gravitational torsional objects". It is worth to stress that also in the case of monopoles in non Abelian Yang-Mills-Higgs theory the Bogomol'nyi equations are linear relations among the magnetic components of the curvature and the covariant derivative of the Higgs field of the type

$$
B^{a}= \pm(D \phi)^{a}
$$

where $B^{a}$ is the non abelian magnetic field. An important point is that often (in this case as well as in the case of monopoles) Bogomol'nyi equations can be formulated as self-duality conditions of a "bigger curvature" of a "bigger connection" in which the gauge connection and the Higgs field fit together [5], [6] in such a way that the self-duality conditions for the bigger curvature are the precisely the above described linear relations among the gauge field strength, the Higgs field and its covariant derivative.

\section{Short Review in Gravity with Torsion}

Here a very short introduction to gravitational actions with torsion will be provided (see also the detailed reviews [3] and [4]).

In the first order formalism the Einstein-Hilbert action in four dimensions can be written as follows:

$$
S_{E H}=\int \varepsilon_{a b c d}\left(\alpha R^{a b} e^{c} e^{d}+\beta e^{a} e^{b} e^{c} e^{d}\right)
$$

where

$$
\begin{array}{rlrl}
e^{a} & =e_{\mu}^{a} \mathrm{~d} x^{\mu}, & R_{b}^{a} q=(\mathrm{d} \omega+\omega \wedge \omega)_{b}^{a} \\
\omega_{b}^{a}=\omega_{b \mu}^{a} \mathrm{~d} x^{\mu}, & g_{\mu \nu}=\eta_{a b} e_{\mu}^{a} e_{\nu}^{b}
\end{array}
$$


$e^{a}$ is the vielbein, $\omega_{b}^{a}$ the spin connection, $g_{\mu \nu}$ is the spacetime metric, $\eta_{a b}$ the Minkowski metric in the vielbein indices and $\alpha$ and $\beta$ are related in a obvious way with the Newton and the cosmological constants. It is manifest in this way that the vielbein indices $a, b, .$. behave as internal gauge indices. Since the Kaluza-Klein idea and with the advent of string theories the possibilities to have extradimensions comes into play. Lovelock [1] showed that it is indeed possible to have gravitational Lagrangian in higher dimensions giving rise to second order equations of motions for the metric, the Lagrangian being of the form

$$
\begin{aligned}
I_{D} & =\kappa \int \sum_{p=0}^{[D / 2]} \alpha_{p} L^{(D, p)} \\
L^{(D, p)} & =\varepsilon_{a_{1} \ldots a_{D}} R^{a_{1} a_{2}} \ldots R^{a_{2 p-1} a_{2 p}} e^{a_{2 p+1}} \ldots e^{a_{D}} .
\end{aligned}
$$

In $D=5$ (on which we will focus from now on) the so called Einstein-GaussBonnet action [1] reads

$$
I=\kappa \int \mathrm{d}^{5} x \sqrt{g}\left(R-2 \Lambda+\alpha\left(R^{2}-4 R_{\mu \nu} R^{\mu \nu}+R_{\alpha \beta \gamma \delta} R^{\alpha \beta \gamma \delta}\right)\right)
$$

where $\kappa$ is related to the Newton constant, $\Lambda$ to the cosmological term, and $\alpha$ is the Gauss-Bonnet coupling ${ }^{1}$. The action (2) in terms of differential forms reads

$$
I=\int \epsilon_{a b c d e}\left(\frac{c_{0}}{5} e^{a} e^{b} e^{c} e^{d} e^{e}+\frac{c_{1}}{3} R^{a b} e^{c} e^{d} e^{e}+c_{2} R^{a b} R^{c d} e^{e}\right) .
$$

The field equations read

$$
\mathcal{E}_{e}:=\left(c_{o} e^{a} e^{b} e^{c} e^{d}+c_{1} R^{a b} e^{c} e^{d}+c_{2} R^{a b} R^{c d}\right) \epsilon_{a b c d e}=0
$$

and

$$
\mathcal{E}_{a b}:=T^{c}\left(c_{1} e^{d} e^{e}+2 c_{2} R^{d e}\right) \epsilon_{a b c d e}=0
$$

while the torsion is

$$
T^{a}=D e^{a} .
$$

In the vanishing torsion sector, the field equations (5) are trivially fulfilled, and equation (4) reduces to the standard one in the second order formalism. An interesting characteristic of the Einstein-Gauss-Bonnet theory is that, unlike in the Einstein-Hilbert case, the torsion is generically different from zero [3] (even if

\footnotetext{
${ }^{1}$ The relationship between the constants appearing in equations (2) and (3) is given by $\alpha=\frac{c_{2}}{2 c_{1}}$, $\Lambda=-6 \frac{c_{0}}{c_{1}}, \kappa=2 c_{1}$.
} 
many authors put torsion equal to zero by hand). Generically, the field equations (5) impose very strong constraints on the torsion (which can be found by taking the covariant derivative of equation (4) and comparing the result with equation (5))

$$
e^{a} R^{b c} T^{d} \epsilon_{a b c d e}=0, \quad e^{a} e^{b} e^{c} T^{d} \epsilon_{a b c d e}
$$

so that, to the best of author's knowledge, no exact solution with torsion has been found in the generic Lovelock case up to recent times. As it will be described in the next section, a nice analogy with BPS states in field theory suggests a natural ansatz for the torsion which solves automatically the constraints in equation (6) allowing to construct exact solutions with torsion in the non-Chern-Simons case [7]. However, there exist special values of the Gauss-Bonnet coupling which enhance the gauge symmetry of the generic Lovelock actions ${ }^{2}$ [3] "killing" the constraints in equation (6)

$$
c_{2}=\frac{c_{1}^{2}}{4 c_{0}}
$$

and leading to the uniqueness of the maximally symmetric vacuum [10]. In this case, a supergravity theory is available and the "BPS form" of the torsion provides one with black hole solutions with a half-BPS ground state [11].

\section{Gauge-Theoretical Interpretation of Torsion}

A useful trick which helps in dealing with torsion in four dimensions is available in the presence of a non vanishing cosmological constant: one can define the following "higher order" connection

$$
W^{A B}=\left[\begin{array}{cc}
\omega^{a b} & -\frac{e^{a}}{l} \\
-\frac{e^{b}}{l} & 0
\end{array}\right]
$$

where $a, b=1, . ., 4$ and $A, B=1, . ., 5$ in such a way that the "higher order" curvature is

$$
F^{A B}=\left[\begin{array}{cc}
R^{a b}-\frac{e^{a} \wedge e^{b}}{l^{2}} & \frac{T^{a}}{l} \\
-\frac{T^{b}}{l} & 0
\end{array}\right]
$$

and the "higher dimensional" topological term reads

$$
F^{A B} \wedge F_{A B}=R^{a b} \wedge R_{a b}+\frac{2}{l^{2}} N .
$$

\footnotetext{
${ }^{2}$ Indeed, for such special values, the Lagrangian can be written as a Chern-Simons form [8]. In the Chern-Simons case, some exact solutions with torsion have been constructed in [9]: however, it seems that in these solutions torsion is pure gauge.
} 
It is worth to stress here that this is also a standard trick in the theory of BPS states which explains why the BPS equations are much simpler than the full YangMills equations: the point is that often one can incorporate the Higgs field and the Yang-Mills connection into a unique higher connection in such a way that the BPS equations are self-duality conditions for the higher curvature (see, for example, [5]). An immediate consequence is that

$$
\int \frac{2}{l^{2}} N=8 \pi^{2} \times n, \quad n \in \mathbb{Z}
$$

that is, two times the integral of the Nieh-Yan density divided by the square of the cosmological length is $8 \pi^{2}$ times an integer (see, for instance, [12]).

The previous trick to incorporate torsion, vielbein and curvature in a "bigger curvature" is standard in studying BPS states in QFT. This analogy suggests that, in gravity, the vielbein and torsion could play the role of the Higgs field and its covariant derivatives in QFT. In other words, one would like to give a precise meaning to the following table

$$
\begin{array}{cc}
\text { Gravity } & \text { Gauge } \\
e^{a} & \phi^{a} \\
T^{b} & (D \phi)^{b} \\
\alpha R^{a b}+\frac{\beta}{2} e^{a} e^{b} & F^{a b}
\end{array}
$$

where $\alpha$ and $\beta$ are suitable constants. In QFT the BPS equations involve typically linear relations (such as higher dimensional self-duality conditions) among $D^{a} \phi$ and $F^{a b}$ in which $\phi$ can enter quadratically (as, for instance, in the vortex case). Thus, a natural ansatz is

$$
T^{c}=f^{c a b}\left(\alpha R^{a b}+\beta e^{a} e^{b}\right)
$$

where $f^{c a b}$ is a three index tensor in the "gravitational gauge group", $\alpha$ and $\beta$ are two constants.

However, a genuine invariant tensor (that is, $f^{a b c}$ in equation (12)) in the Lorentz group does not exist in general. As it is well known (see, for instance, [13]) some breaking of the vacuum symmetries is needed. In this case, a tensor $f^{a b c}$ which makes consistent equation (12) can be constructed using the broken generator(s). The simplest invariant tensor with three indices is the Levi-Civita symbol $\varepsilon^{a b c}$. In the five-dimensional case (which has been analyzed in [7] and [11]) this corresponds to consider solutions which are a direct or a warped product of a two 
dimensional manifold $M_{2}$ and a three dimensional manifold $M_{3}$ of constant curvature on which the torsion lives

$$
T^{c}=K\left(x^{1}, x^{2}\right) \varepsilon^{c a b} e^{a} e^{b}
$$

where $K$ may depend on the coordinates $\left(x^{1}, x^{2}\right)$ of $M_{2}$ and the vielbeins $e^{a}$ and the indices $a, b$ and $c$ refer to $M_{3}$. Remarkably enough, this ansatz automatically solves both constraints in equation (6).

\subsection{The Non-Chern-Simons Case}

For the first time, the above ansatz (13) allowed to find exact vacuum solutions with torsion in five dimensional Einstein-Gauss-Bonnet gravity for a suitable choice of the constants appearing in the action which does not correspond to a ChernSimons theory [7]. The metric is given by

$$
\mathrm{d} s^{2}=\mathrm{d} s_{\mathrm{AdS}_{2}}^{2}+\mathrm{d} s_{\mathrm{S}^{3}}^{2} .
$$

The torsion is introduced onto the $M_{3}$ in such a way as to respect the symmetry:

$$
T^{a}=\tau \epsilon_{a b c} e^{b} e^{c}
$$

By imposing the relation

$$
c_{1}^{2}=12 c_{0} c_{2}
$$

(which, interestingly enough, does not correspond to the Chern-Simons case) one gets an exact vacuum solution with torsion in Einstein-Gauss-Bonnet gravity whose curvatures are

$$
\begin{gathered}
T_{c}= \pm \sqrt{\frac{1}{D^{2}}+\frac{1}{3 l^{2}}} \epsilon_{a b c} e^{b} e^{c} \\
R^{01}=-\frac{1}{l^{2}} e^{0} e^{1}, \quad R^{a b}=-\frac{1}{3 l^{2}} e^{a} e^{b}
\end{gathered}
$$

provided that $c_{2} / c_{1}$ and $c_{0} / c_{1}$ are positive. It is worth to note that the torsion is bounded from below for finite sized sphere and AdS length scale. This means that there is no continuous zero torsion limit.

Furthermore, if one defines the Hodge dual of the three form $T$

$$
\begin{aligned}
T & \equiv T_{a b c} e^{a} \wedge e^{b} \wedge e^{c}=3 ! \tau e^{2} \wedge e^{3} \wedge e^{4} \\
* T & =-2 ! \tau e^{0} \wedge e^{1}
\end{aligned}
$$


then

$$
D T=0, \quad D * T=0
$$

so that, by defining $F \equiv * T, F$ is seen to obey the source-free Maxwell equations, making manifest the close resemblance with the electromagnetic field of the Bertotti-Robinson solution [14].

\subsection{The Chern-Simons Case}

Let us now shortly describe the Chern-Simons case in which

$$
c_{1}^{2}=4 c_{0} c_{2}
$$

In the case of the five-dimensional Chern-Simons gravity, spherically symmetric black hole without torsion are well known

$$
\mathrm{d} s^{2}=-\left(\frac{r^{2}}{l^{2}}-\mu\right) \mathrm{d} t^{2}+\frac{\mathrm{d} r^{2}}{\frac{r^{2}}{l^{2}}-\mu}+r^{2} \mathrm{~d} \Sigma_{3}^{2}
$$

and are quite similar to three-dimensional BTZ black-hole [15], [16]. In both cases, when the mass parameter $\mu$ is $\mu=-1$ the solution is $\operatorname{AdS}_{3}$ and $\mathrm{AdS}_{5}$. When the mass parameter is greater than zero one has a black hole and when $-1<\mu<0$, timelike naked singularities appear. When torsion vanishes and without matter field, in the five dimensional case the zero mass black hole breaks all the supersymmetries [17]. While in three dimensions the $\mu=0$ is the half-BPS "black hole vacuum". If torsion is introduced in $\Sigma_{3}$ as follows

$$
T^{a}=-\frac{\delta}{r} \epsilon^{a b c} e_{b} e_{c}
$$

the above metric still represents a five-dimensional black hole [11]. Furthermore, when the constant $\delta$ is equal to one (or minus one), it can be shown that the solution with $\mu=0$ is half-BPS exactly in the same way as it happens in three dimensions (something impossible without torsion). Thus, torsion improves the stability analysis of the theory.

\subsection{A Physical Interpretation}

The nice features of the results in [7] and [11] (which have been resumed in the previous subsections) is that in five (and, presumably, higher dimensions) one can construct with a very natural recipe exact vacuum solutions with non-trivial geometrical structures which, in four dimensions, could only be obtained coupling 
gravity to suitable matter fields. The reason is that, being the total connection the sum of the Riemannian part plus the contorsion, one can manage to choose the contorsion in such a way to exactly cancel (a suitable part of) the purely Riemannian connection (as it has been done in [11]). This is quite similar to what happens, for instance, in the case of the extreme charged black-hole in Einstein gravity: the electromagnetic connection cancels part of the Levi-Civita connection leading to an half-BPS solution. This means that, in higher dimensions, the gravitational interaction is much more complex allowing, in some cases, to "emulate" matter fields much more effectively than in Einstein gravity: in other words, in higher dimensions gravity can be more "repulsive" than in four dimensions providing one with the possibility to shed new light on some of the open question in cosmology. One can see this as follows: from a particles physics perspective, the Riemannian part of the gravitational interactions can be represented by a spintwo particle, the graviton, which generates basically an attractive interaction. In four dimensions, this is the only possibility compatible with the requirements of general covariance and with second order equations of motion for the metric. In higher dimensions torsion is not zero anymore so that also a non-Riemannian part of the gravitational field appears. In many cases, the torsion generates a repulsive interaction: for instance, in the previous subsections it has been shown that torsion may behave as an electromagnetic field generating in vacuum a BertottiRobinson-like solution. Furthermore, torsion may also stabilize the spherically symmetric Chern-Simons black-hole preventing a decay into naked singularities. For this reason, the role of torsion is worth to be further investigated.

\section{Observable Effects}

In this section some observable effects in high energy physics and solid state physics related to the above discussion will be analyzed.

\subsection{High Energy Physics}

If the Einstein-Hilbert Lagrangian is the reduction of some higher dimensional model (indeed, Chern-Simons theories are very natural candidates [18]) the torsion generically does not vanish and propagates [19]. One should consider the possibility that somewhere in the universe torsion could be present. Let us consider the case of Chern-Simons theory (related to the (AdS vacuum) in which torsion is non zero only along two direction (say, 1 and 2). One can consider four dimensional cosmological models of Einstein equations as coming from a suitable 
Chern-Simons solutions.

An interesting effect of non vanishing torsion is related to neutrinos oscillations. In [20] it has been shown that the torsion can contribute to neutrino oscillation along a path of length $\Delta r$ (among neutrinos of different polarization)

$$
(\Delta \Phi)_{T}=\Phi^{+}-\Phi^{-} \approx \frac{1}{l^{\prime}} \Delta r
$$

where the + and the - refer to the up and down polarizations, $l$ is the coefficient in front of the torsion term in the covariant derivative. The contribution of torsion to oscillations can be understood because for Fermion, in rough terms, the coupling with torsion is similar to the coupling with mass. The authors of [20] took as $l^{\prime}$ the Planck length (arguing that the torsion, being a quantum effects, should appear at the Planck scale): the result was that the contribution of the torsion is 55 order of magnitude less than the field theoretical one $(\Delta \Phi)_{Q F T}$

$$
\begin{aligned}
(\Delta \Phi)_{Q F T} & \approx \frac{c^{4} \Delta m^{2}}{2 E \hbar c} \Delta r \\
\frac{c^{4} \Delta m^{2}}{2 E \hbar c} & \approx 10^{-9}[m]^{-1} .
\end{aligned}
$$

It is much more natural from the theoretical point of view (see, for instance, [3] and [4]) that the constant $l^{\prime}$ is related to the cosmological constant. In such a case, the contribution of the torsion is six order of magnitude larger then the observed one. Thus, in this case also it seems not possible to explain neutrino oscillations with torsion.

It cannot be excluded that in a more refined setting one could get an order of magnitude closer to the observed one. The results of [20] follow from two assumptions: the first is that "the torsion coupling constant" is the Planck length ${ }^{3}$. The second is that the torsion is related to the Dirac fields describing the neutrinos as dictated by the Einstein equations and, moreover, it is assumed that the torsion has not a dramatic dependence on the spacetime coordinates ${ }^{4}$ so that, in order to estimate the effects of the torsion it is enough to take into account the order of magnitude of the torsion coupling constant. It has been shown that there exist solutions in five dimensional Chern-Simons theory in which one has non trivial torsion along some directions and that a non trivial spacetime dependence is possible. In the present formalism (assuming for simplicity that there is only one

\footnotetext{
${ }^{3}$ It has been already discussed that it is almost mandatory from the geometric point of view to assume that "the torsion coupling constant" is the cosmological length.

${ }^{4}$ In practice, in [20] the possible spacetime dependence of the torsion is neglected.
} 
deformed tetrad field), the phase factor arising in equation (18) of [20] is related to the integral

$$
\frac{1}{l} \int_{\gamma} \delta e_{\mu}^{1} \mathrm{~d} x^{\mu}
$$

where $\delta e_{\mu}^{1}$ is the deformation of tetrad field responsible of the non vanishing of the torsion and $\gamma$ is a spacelike geodesic joining the two points among which one wants to compute the oscillations. One obtains the results of [20] in the case in which there is no dependence on the coordinates. However, at this stage of the analysis it is not clear how to determine the torsion from first principles since in Chern-Simons theory there is a huge freedom allowing for too many different deformations of the tetrad.

\subsection{Solid State Physics}

In the theory of elasticity the analysis of defects can be conveniently formulated (see [21-24]) by describing the medium as a continuum geometry with localized torsion. In particular, torsion singularities located on parallel lines appears to be well suited to describe dislocations [21], [22] while the geometry of cosmic strings can describe rather well [24] the disclinations present in various type of materials. The Dirac equation in presence of torsion can be written as follows

$$
\mathrm{i} \Gamma^{\mu}\left(\partial_{\mu}-\frac{\omega_{\mu}^{a b}}{4} \Gamma_{a b}+\frac{1}{2 l} e_{\mu}^{a}\left((\mathrm{i})^{n} \Gamma_{a}\right)\right) \Psi(x)=0
$$

where $\omega_{\mu}^{a b}$ is the Levi-Civita connection, $\Gamma^{a}$ are the flat Dirac matrices, $\Gamma^{\mu}$ the curved ones $^{5}, \Psi$ is the Dirac spinor, $l$ is a length scale and $n$ can be zero or one (as it will be discussed in a moment together with the physical meaning of length scale $l$ ). The added term is quite natural in a curved spacetime and should not be neglected. When dealing with standard gravitational models, it can be gauged away

$$
\begin{aligned}
\partial_{\mu}-\frac{\omega_{\mu}^{a b}}{4} \Gamma_{a b}+\frac{1}{2 l} e_{\mu}^{a}\left((\mathrm{i})^{n} \Gamma_{a}\right) & \rightarrow \partial_{\mu}-\frac{\omega_{\mu}^{a b}}{4} \Gamma_{a b} \\
\Psi(x) & \rightarrow P \exp \left[\mathrm{i} \int^{x} e_{\mu}^{a}(y)\left((\mathrm{i})^{n} \Gamma_{a}\right) \mathrm{d} y^{\mu}\right] \Psi(x)
\end{aligned}
$$

\footnotetext{
${ }^{5}$ The above Dirac equation is an effective equation because it can only describe the low energy electronic excitations near the Fermi surface. Once the propagator is known, it is possible to compute the electronic density around defects.
} 
where $P$ is the path ordering. The integral in the phase factor does not depend on the path because the covariant derivative of the tetrad $e_{\mu}^{a}$ vanish and therefore it has no physical effects. In the presence of dislocations this is not true anymore since the torsion does not vanish. When the torsion is localized along the $z$ axis the Aharonov-Bohm phase factor $\Phi$ is

$$
\Phi=\exp \left[\frac{\mathrm{i}}{2 l} \iint \mathrm{d} x \mathrm{~d} y T\right]
$$

The integral in the exponent has a very nice physical interpretation: it is the so called Burgers vector $\vec{b}$ which is proportional to the integral of the flux of the torsion through a surface [21]

$$
\iint_{S} \mathrm{~d} x^{m} \wedge \mathrm{d} x^{n} T_{m n}^{i}=-b^{i} .
$$

Therefore, the length scale $l$ in a solid state physics context has to be interpreted as the mean "size" of the Burgers vector

$$
2 l \sim|\vec{b}| .
$$

Such a vector is very important to describe the physical effects of edge dislocations which are rather common in nature ${ }^{6}$. Another interesting possibility has been proposed in [25] in which topological defects manifest themselves through anomalous conductivities.

The quantum effects of dislocations can manifest themselves also by computing the propagator in an effective three dimensional gravitational field with torsion localized along some spacelike line. One can think to a three dimensional piece of material in which the interesting phenomena occur in a two dimensional planes orthogonal to the dislocation. Let us consider the effective Dirac equation equation (19) describing the low energy electronic excitations in the field of a "vortex of torsion" along the $z$ axis (but without other defects near the dislocation)

$$
\mathrm{i} \Gamma^{\mu}\left(v_{F} \partial_{\mu}+\frac{(\mathrm{i})^{n}}{2 l} e_{\mu}^{a}\left(\Gamma_{a}\right)\right) \Psi(x)=0
$$

\footnotetext{
"Pictorially" such defects arise when one "cuts" some pieces of material and then glue the boundary in a suitable way with or without adding extra pieces of material obtaining a much higher or a much lower density. Non vanishing torsion can describe both wedge dislocations (which are rather uncommon) and edge dislocations (which are often encountered in practical applications) [23].
} 
where $v_{F}$ is the Fermi velocity of the material (which, henceforth will be set equal to one). To proceed, one has to assume that the "torsional" term can be treated as a small perturbation ${ }^{7}$.

Besides the obvious term, the tetrad fields and the curved metric also manifest themselves through the curved Dirac matrices. In the present case, one is analyzing an effective two-dimensional situation in which only the third tetrad field is deformed in such a way to achieve a "vortex of torsion" along the $z$-axis and the meaningful dynamics is in the $x-y$ plane so that the explicit dependence on $z$ of the Dirac wave function can be neglected. The structure of the spatial tetrad fields is

$$
e^{1}=\mathrm{d} x, \quad e^{2}=\mathrm{d} y, \quad e^{3}=\mathrm{d} z+a_{i} \mathrm{~d} x^{i}, \quad i=1,2=x, y
$$

(where the explicit form of $a_{1}$ and $a_{2}$ is not important in this moment) so that the inverse tetrads read

$$
\begin{array}{ll}
e_{a}=e_{a}^{(0)}+\delta e_{a}^{(0)}, & e_{3}=\partial_{z} \\
e_{1}=\partial_{x}-a_{1} \partial_{z}, & e_{2}=\partial_{y}-a_{2} \partial_{z} .
\end{array}
$$

The further terms $\delta\left(\Gamma^{\mu} \partial_{\mu}\right)$ in equation (21) coming from the curved Dirac matrices are

$$
\delta\left(\Gamma^{\mu} \partial_{\mu}\right)=-\left(a_{1} \Gamma^{1}+a_{2} \Gamma^{2}\right) \partial_{z}
$$

In the approximation in which the effective dynamics is two dimensional, the derivative with respect to $z$ can be neglected. Therefore, the only further term in equation (21) with respect to a flat Dirac equation is the one in which it explicitly appears the tetrad field multiplied by $\frac{(\mathrm{i})^{n}}{2 l}$.

Thus, when the effective dynamics is two-dimensional (so that the dependence on $z$ can be neglected), the effect of dislocations can be described in exactly the same way as the effects of disclinations. Thus, for instance, if one manages to compute the total density of states $\rho(\omega)$ of the electrons localized near a disclination then one can use the same formalism to compute the density of electrons near a dislocation. In order to proceed in the computation, one needs the Fourier transform of $\delta e_{\mu}^{3}$ (which, in the present geometric perspective, is the "source of torsion"). Dislocations can be described as torsion localized along some axis (say, the $z$ axis). In the case in which the geometry is locally flat besides the dislocation itself, the suitable form of $\Gamma_{3} \delta e_{i}^{3}$ able to describe torsion localized along the $z$ axis is formally the same as the one needed to describe a thin magnetic flux along the

\footnotetext{
${ }^{7}$ In the case of the defects analyzed in [24] such approximation gives results in very good agreement with observations.
} 
same axis

$$
\frac{\Gamma_{3} \delta e_{j}^{3}}{2 l}=A_{j}^{\text {Vort }}=\frac{\Theta}{2 \pi} \varepsilon_{3 j k} \frac{x^{k}}{r^{2}}
$$

where $\Theta$ represents the strength of the torsion (which, as it has been already stressed, is proportional to the modulus of the Burgers vector)

$$
\Theta=\int_{\gamma} A_{j}^{V o r t} \mathrm{~d} x^{j} .
$$

Here $\gamma$ is a closed curve around the origin in the $x-y$ plane. After this identification the computations to obtain the electron density near the defect may follow the line of $[24]^{8}$.

Because of its reach geometrical structure, the phenomenological role of torsion in solid state physics is worth to be further investigated.

\section{Conclusion}

It has been shown that in higher dimensional gravitational Chern-Simons theories there exist solutions with non vanishing torsion which rapidly approaches to zero outside suitable spacetime regions. The possible contribution of the torsion to neutrino oscillations has been already discussed. However, this formalism clarifies that if one correctly takes into account the geometric nature of torsion the contribution of torsion to neutrino oscillations could be rather closer to the observed value. This scheme has also interesting applications in solid state physics. Dislocations (rather common defects in solid state physics) could be observable both via Aharonov-Bohm type experiments.

\section{Acknowledgements}

The author would like to thank J. Zanelli for many suggestions and continuous encouraging, Andres Anabalon, Alex Giacomini, Ricardo Troncoso and Steven Willison for many stimulating discussions and important bibliographic suggestions. This work was supported by Fondecyt grant 3070055. The Centro de Estudios Científicos (CECS) is funded by the Chilean Government through the Millennium Science Initiative and the Centers of Excellence Base Financing Program of

\footnotetext{
${ }^{8}$ In which, however, there is a mistake. I thank M. A. H. Vozmediano for pointing out this to me. However, the important point of the present discussion is that in principle one can get the effects of dislocations in two space dimensions from the analogous computation for a disclination. Thus, the technical mistake in [24] does not affect this possibility.
} 
Conicyt. CECS is also supported by a group of private companies which at present includes Antofagasta Minerals, Arauco, Empresas CMPC, Indura, Naviera Ultragas and Telefónica del Sur. CIN is funded by Conicyt and the Gobierno Regional de Los Ríos.

\section{References}

[1] Lovelock D., The Einstein Tensor and its Generalizations, J. Math. Phys.12 (1971) 498-501.

[2] Garraffo C. and Giribet G., The Lovelock Black Holes, arXiv:0805.3575.

[3] Troncoso R. and Zanelli J., Gauge Supergravities for All Odd Dimensions, Int. J. Theor. Phys. 38 (1999) 1181-1206; Higher Dimensional Gravity, Propagating Torsion and AdS Gauge Invariance, Quant. Grav. 17 (2000) 4451-4466.

[4] Zanelli J., Lecture Notes on Chern-Simons (Super-Gravities, hepth/0502193.

[5] Olive D. and West P. (Eds), Duality and Supersymmetric Theories, Cambridge University Press, Cambridge, 1999.

[6] Tong D., TASI Lectures on Solitons, hep-th/0509216.

[7] Canfora F., Giacomini A. and Willison S., Some Exact Solutions with Torsion in 5-D Einstein-Gauss-Bonnet Gravity, Phys. Rev. D 76 (2007) 044021.

[8] Chamseddine A., Topological Gauge Theory Of Gravity In Five-Dimensions and All Odd Dimensions, Phys. Lett. B 233 (1989) 291-294.

[9] Aros R. and Contreras M., Torsion Induces Gravity, Phys. Rev. D 73 (2006) 087501.

[10] Crisostomo J., Troncoso R. and Zanelli J., Black Hole Scan, Phys. Rev. D 62 (2000) 084013.

[11] Canfora F., Giacomini A. and Troncoso R., Black Holes, Parallelizable Horizons and Half-BPS States for the Einstein-Gauss-Bonnet Theory in Five Dimensions, Phys. Rev. D 77 (2008) 024002.

[12] Chandia O. and Zanelli J., Supersymmetric Particle in a Space-Time with Torsion and the Index Theorem, Phys. Rev. D 55 (1998) 045014.

[13] Weinberg S., The Quantum Theory of Fields, vols I\&II, Cambridge University Press, Cambridge, 1996.

[14] Bertotti B., Uniform Electromagnetic Field in the Theory of General Relativity, Phys. Rev. 116 (1959) 1331-1333; Robinson I., Bull. Acad. Pol. Sci., Ser. Sci. Phys. Astron. 7 (1959) 351. 
[15] Banados M., Teitelboim C. and Zanelli J., The Black Hole in ThreeDimensional Space-Time, Phys. Rev. Lett. 69 (1992) 1849-1851.

[16] Banados M., Henneaux M., Teitelboim C. and Zanelli J., Geometry of the (2+1) Black Hole, Phys. Rev. D 48 (1993) 1506-1525.

[17] Aros R., Martinez C., Troncoso R. and Zanelli J., Supersymmetry of Gravitational Ground States, J. High Energy Phys. 05 (2002) 020.

[18] Anabalon A., Willison S. and Zanelli J., General Relativity from a Gauged WZW Term, Phys. Rev. D 75 (2007) 024009.

[19] Banados M., Garay L. and Henneaux M., The Local Degrees of Freedom of Higher Dimensional Pure Chern-Simons Theories, Phys.Rev.D 53 (1996) 593; The Dynamical Structure of Higher Dimensional Chern-Simons Theory, Nucl. Phys. B 476 (1996) 611-635.

[20] Adak M., Dereli T. and Ryder L., Neutrino Oscillations Induced by SpaceTime Torsion, Class. Quant. Grav. 18 (2001) 1503-1512.

[21] Katanaev M. and Volovich I., Theory of Defects in Solids and ThreeDimensional Gravity, Ann. Phys. 216 (1992) 1-28.

[22] Frohlich J. and Studer U., Gauge Invariance and Current Algebra in Nonrelativistic Many-Body Theory, Rev. Mod. Phys. 65 (1993) 733-802.

[23] Katanaev M., Geometric Theory of Defects, cond-mat/0407469; Introduction to the Geometric Theory of Defects, cond-mat/0502123.

[24] Cortijo A. and Vozmediano M., Effects of Topological Defects and Local Curvature on the Electronic Properties of Planar Graphene, Nucl. Phys. B 763 (2007) 293-308.

[25] Balachandran A., John V., Momen A. and Moraes F., Anomalous Defects and Their Quantized Conductivities, Int. J. Mod. Phys. A 13 (1998) 841861.

Fabrizio Canfora

Area de Fisica Teorica

Centro de Estudios Cientificos (CECS)

Valdivia

CHILE

E-mail address: canforaececs.cl 